\title{
Quasicanonical Gibbs distribution and Tsallis nonextensive statistics
}

\author{
A.K. Aringazin and M.I. Mazhitov \\ Department of Theoretical Physics, \\ Institute for Basic Research, Eurasian National University, \\ Astana 473021 Kazakstan
}

17 April 2002

\begin{abstract}
We derive and study quasicanonical Gibbs distribution function which is characterized by the thermostat with finite number of particles (quasithermostat). We show that this naturally leads to Tsallis nonextensive statistics and thermodynamics, with Tsallis parameter $q$ is found to be related to the number of particles in the quasithermostat. We show that the chi-square distribution of fluctuating temperature used recently by Beck can be partially understood in terms of normal random momenta of particles in the quasithermostat. Also, we discuss on the importance of the time scale hierarchy and fluctuating probability distribution functions in understanding of Tsallis distribution, within the framework of kinetics of dilute gas and weakly inhomogeneous systems.
\end{abstract}

\section{Introduction}

Nonextensive statistical mechanics introduced by Tsallis [1] has been developed and applied by many authors [2]-[8]; see [3] for a recent review. Particularly, various aspects of the nonextensive statistics and thermodynamics have been studied by Abe and Rajagopal 团. Derivation of power law canonical distributions from first principles of statistical mechanics has been analyzed by Almeida [9]; see also [10]. 
The entropic parameter $q$ of Tsallis statistics is associated to the measure of nonextensivity, with the usual extensivity recovered at $q=1$. In Tsallis formalism, $q$ appears in the definition of entropy which generalizes the usual Boltzmann-Gibbs-Shannon entropy.

In this paper we show that a nonextensivity characterized by a parameter naturally arises from the consideration of the microcanonical Gibbs distribution. Namely, in Sec. 2 we consider a subsystem weakly interacting with a system containing big but finite number $M$ of particles which we call quasithermostat. It appears that the resulting equilibrium distribution function and the associated entropy (Sec. 3) reproduce exactly those of Tsallis statistics, with $q-1 \sim 1 / M$. In the (thermodynamic) limit, $M \rightarrow \infty$, the usual canonical Gibbs distribution is recovered.

Recently, Beck [5] have studied application of Tsallis formalism to turbulent flows and achieved a very good agreement with experimental measurements. Particularly, it is worthwhile to emphasize that no fitting parameters have been used by Beck to reproduce histogram of the acceleration of a test particle advected by the turbulent flow, while some other authors have used four free parameters in some ad hoc modified exponential function, for this purpose (see [5] for details). The main idea underlying Tsallis statistical mechanics approach to turbulence is to introduce fluctuation of temperature or fluctuation of energy dissipation [7, 8] described by $\chi^{2}$ distribution of order $m$.

In this paper we show that the relevance of $\chi^{2}$ distribution can be understood in terms of normal random momenta of particles in the quasithermostat, with $m=3 M$ representing the total number of random variables (Sec. 4).

Some aspects of fluctuations and time scale hierarchy in dilute gas and weak inhomogeneous systems are presented in Sec. 1 .

In this paper we show the importance of the time scale hierarchy and fluctuating probability distribution functions in understanding Tsallis distribution, which is viewed as the average value of fluctuating canonical Gibbs distribution over the random parameter $\tilde{\beta}$.

\section{Quasicanonical Gibbs distribution}

We start with the classical microcanonical Gibbs distribution, $f_{L}(x, a, E)$, which describes isolated equilibrium system of $L$ classical particles with total 
energy $E$,

$$
f_{L}(x, a, E)=\frac{1}{\Omega(a, E)} \delta(H(x, a)-E),
$$

where $\Omega(a, E)=\int \delta(H(x, a)-E) d x, H(x, a)$ is Hamiltonian of the system, $a$ denotes external parameters, $d x=\prod_{1 \leq i \leq L} d x_{i}$, and $x_{i}=\left(\vec{p}_{i}, \vec{r}_{i}\right)$ are phasespace coordinates of the particles.

In the closed system of $L$ particles we select a subsystem of $N \gg 1$ particles which is surrounded by the subsystem of $L-N=M$ particles. Assuming $M \gg N$, neglecting the interaction energy between the subsystems, and taking the limit $M \rightarrow \infty$, from the microcanonical distribution (1) one can derive, following one of the known procedures, the usual canonical Gibbs distribution,

$$
f_{N}(x, a, T)=\frac{1}{Z} \exp \left[-\frac{H_{N}(x, a)}{k T}\right],
$$

where $Z=\int \exp \left[-H_{N}(x, a) / k T\right] d x$ is the classical statistical integral. Here, $k$ is Boltzmann constant and $T$ is an absolute temperature of the subsystem of $M=\infty$ particles (called thermostat); $d x=\prod_{1 \leq i \leq N} d x_{i}$.

It is worthwhile to note that the canonical Gibbs distribution comes also as the only physically relevant solution of supersymmetry condition in the context of topological quantum field theory approach to Hamiltonian systems [11].

Below, we follow some procedure assuming that the number of particles in thermostat is finite, $M<\infty$. Such a thermostat can be referred to as a quasithermostat.

The total Hamiltonian is represented by

$$
H(x, y)=H_{N}(x)+H_{M}(y)+H_{\text {int }}(x, y),
$$

where $x$ and $y$ denote phase-space coordinates of particles in the considered subsystem and in the quasithermostat, respectively, and $H_{\text {int }}(x, y)$ characterizes interaction between the subsystem and quasithermostat. Herebelow, we drop dependence on the external parameters to simplify notation.

Since the total system is closed, it is described by the microcanonical distribution,

$$
f_{N+M}(x, y, E)=C \delta(H(x, y)-E),
$$

provided that it is normalized, $\int f_{N+M}(x, y, E) d x d y=1 ; d y=\prod_{1 \leq i \leq M} d y_{i}$. Note that the numbers $M$ and $N$ are additive integrals of motion. To obtain 
the distribution function describing the considered subsystem one should integrate out phase-space coordinates $y$ of the quasithermostat,

$$
f_{N}(x)=C \int \delta\left(E-H_{N}(x)-H_{M}(y)-H_{\text {int }}(x, y)\right) d y,
$$

For big $N$ and weak short-range interactions one can ignore the interaction energy, $H_{\text {int }}(x, y) \ll H_{N}(x)$, in the above expression. By representing Hamiltonian of the quasithermostat as a sum of the kinetic and potential energy,

$$
H_{M}(y)=K_{M}\left(\vec{p}_{1}, \ldots, \vec{p}_{M}\right)+U_{M}\left(\vec{r}_{1}, \ldots, \vec{r}_{M}\right),
$$

where we take for simplicity

$$
K_{M}(p)=\sum_{i=1}^{M} \frac{\vec{p}_{i}^{2}}{2 m}
$$

and $U_{M}(r)=\sum_{i \neq j} \Phi\left(\left|\vec{r}_{i}-\vec{r}_{j}\right|\right)$ for point-like particles of mass $m$, we can rewrite (5) as

$$
f_{N}(x)=C \int \delta\left(E-H_{N}(x)-U_{M}(r)-z^{2}\right) \delta\left(z-K_{M}^{1 / 2}\right) d z d p d r
$$

where new variable $z$ has been used; $d p=\prod_{1 \leq i \leq M} d \vec{p}_{i}, d r=\prod_{1 \leq i \leq M} d \vec{r}_{i}$. Performing the integral over $z$ with the use of

$$
\int \delta\left(z-K_{M}^{1 / 2}(p)\right) d p \simeq z^{3 M-1}
$$

and general property of Dirac delta function,

$$
\int \delta(g(z)) d z=\int \sum_{k} \frac{\delta\left(z-z_{k}\right)}{\left|g^{\prime}\left(z_{k}\right)\right|} d z, \quad g\left(z_{k}\right)=0,
$$

we get, up to normalization constant,

$$
f_{N}(x)=C \int\left(E-H_{N}(x)-U_{M}(r)\right)^{\frac{3 M}{2}-1} d r .
$$

We rearrange this as follows

$$
f_{N}(x)=C \int\left(1-\frac{H(x)}{E-U_{M}(r)}\right)^{\frac{3 M}{2}-1} \times
$$




$$
\times\left(E-U_{M}(r)\right)^{\frac{3 M}{2}-1} d r
$$

where we have denoted, for brevity, $H(x) \equiv H_{N}(x)$.

Denoting the kinetic energy per particle by

$$
\frac{3}{2 \beta}=\frac{K_{M}(p)}{M}
$$

which tends to constant for big $M$, we obtain that for $M \gg N$,

$$
E-U_{M}(r) \simeq \frac{3 M}{2 \beta}
$$

which does not depend on $r$.

Inserting (14) into (12) and performing the integral over $r$ we obtain, up to normalization constant,

$$
f_{N}(x)=C\left(1-\frac{2}{3 M} \beta H(x)\right)^{\frac{3 M}{2}-1} .
$$

We note that to obtain the canonical Gibbs distribution (2) it remains to take the (thermodynamic) limit,

$$
\lim _{M \rightarrow \infty}\left(1-\frac{2}{3 M} \beta H(x)\right)^{\frac{3 M}{2}-1}=\exp [-\beta H(x)]
$$

and identify $\beta^{-1}=k T$.

However, we are interested specifically in the case when $M$ is finite so that we do not take the above (thermodynamic) limit, and turn to analysis of the distribution function (15).

For $M \gg 1$ we make the approximation $3 M / 2-1 \simeq 3 M / 2$. Then, denoting

$$
q-1=\frac{2}{3 M} \geq 0
$$

we have from (15)

$$
\begin{array}{r}
f_{N}(x, a)=\frac{1}{Z_{q}} \\
(1-(q-1) \beta H(x, a))^{\frac{1}{q-1}} \\
\equiv \frac{1}{Z_{q}} \exp _{q-1}[-\beta H(x, a)] .
\end{array}
$$


Here, we have introduced $Z_{q}=\int \exp _{q}[-\beta H(x, a)] d x$ due to the normalization condition, $\int f_{N} d x=1$, q-exponential is defined by $\exp _{s}[z]=(1+s z)^{1 / s}$, $s=q-1$, and we have restored dependence on the external parameters $a$. In view of the fact that the number $M$ is taken to be big but finite, i.e. $q \simeq 1$, the normalized distribution function (18) can be referred to as the quasicanonical Gibbs distribution.

The obtained distribution function (18) strongly resembles the distribution function in Tsallis nonextensive statistics [1, 2, 3], where $q$ is called entropic parameter.

We remind that $q$-exponential, $\exp _{q-1}[z]$, reduces to the ordinary exponential, $\exp [z]$, at $q \rightarrow 1$, so that the canonical Gibbs distribution is recovered.

Typical plots of $\exp _{q-1}[-z]$ at various $q$ are presented in Fig. 1. In the physical context, to avoid the negative and increasing values of $\exp _{q-1}[-z]$ one must restrict positive $z$ by a suitable condition (the high-energy cut-off).

Some useful formulas with $q$-exponential are:

$$
\begin{gathered}
d \exp _{q-1}[ \pm z] / d z= \pm\left(\exp _{q-1}[ \pm z]\right)^{2-q} \\
\int \exp _{q-1}[ \pm z] d z= \pm q^{-1}\left(\exp _{q-1}[ \pm z]\right)^{q} \\
\exp _{q-1}[-z]=\left(\exp _{1-q}[z]\right)^{-1}, \\
\exp _{q-1}\left[z_{1}\right] \exp _{q-1}\left[z_{2}\right]=\exp _{q-1}\left[z_{1}+z_{2}+(q-1) z_{1} z_{2}\right]
\end{gathered}
$$

the $q$-Poisson integral,

$$
\int z^{n} e_{q-1}^{-c z^{2}} d z=z^{n+1}{ }_{2} F_{1}\left(\frac{n+1}{2}, \frac{1}{1-q}, \frac{n+3}{2},(q-1) c z^{2}\right),
$$

and

$$
\begin{gathered}
\int_{0}^{\infty} z^{n} e_{q-1}^{-c z^{2}} d z=\frac{1}{2}(c-c q)^{-(n+1) / 2} \times \\
\times \Gamma\left(\frac{n+1}{2}\right) \Gamma\left(\frac{q+n(q-1)+1}{2(1-q)}\right) \Gamma^{-1}\left(\frac{1}{1-q}\right),
\end{gathered}
$$

for $n>-1, \frac{q+n(q-1)+1}{2(1-q)}>0, \frac{n+1}{2}-\frac{1}{1-q}>0$, and real $c$, where ${ }_{2} F_{1}$ is hypergeometric function and $\Gamma$ is Euler gamma function.

The reflection of the $q$-axis with respect to the point $q=1$, maps $\exp _{q-1}[z]$ to $\exp _{1-q}[z]=(1+(1-q) z)^{1 /(1-q)}$ which is often used in the literature as a definition of the $q$-exponential. 


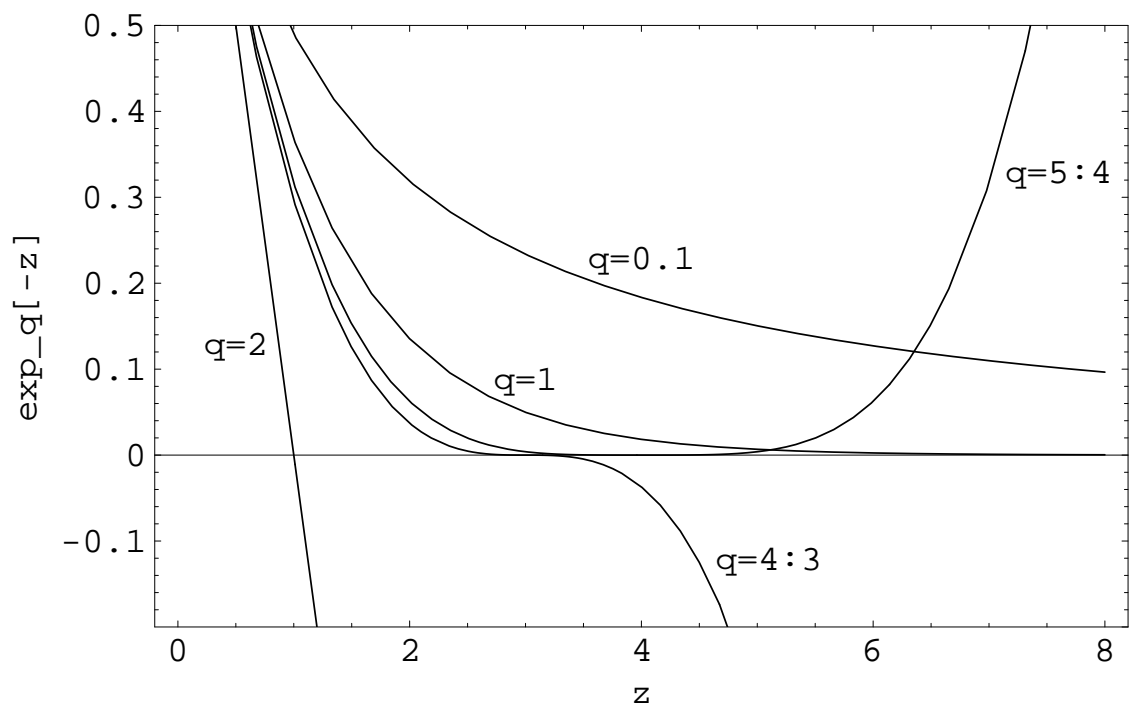

Figure 1: The $q$-exponential $\exp _{q-1}[-z]=(1-(q-1) z)^{1 /(q-1)}$ at various values of $q$.

Also, it is remarkable to note that the slightly generalized $q$-exponential,

$$
\exp _{q-1, b}[z] \equiv(1+(q-1) z)^{1 /(q-1)+b}
$$

can be represented as a power of the $q$-exponential,

$$
(1+(q-1) z)^{c /(q-1)}=\left(\exp _{q-1}[z]\right)^{c},
$$

for the parameter $c=1+(q-1) b$. For example, $(1+(q-1) z)^{1 /(q-1)+1}=$ $(1+(q-1) z)^{q /(q-1)}=\left(\exp _{q-1}[z]\right)^{q}$, so that for small $q-1$ we have $\exp _{q-1}[z] \simeq$ $\left(\exp _{q-1}[z]\right)^{q}$.

Note that $\left(\exp _{q-1}[z]\right)^{c}$ corresponds to the scaling of $z$ in the $q \rightarrow 1$ limit, namely, $\left(\exp _{q-1}[z]\right)^{c} \rightarrow \exp [c z]$, while in general $\left(\exp _{q-1}[z]\right)^{c} \neq \exp _{q-1}[c z]$.

In contrast to the canonical case, the distribution function (18) depends not only on the temperature $\left(\beta^{-1}\right)$ but also on the total number of particles in quasithermostat displayed by the parameter $q$. Also, the property (22) of $q$ exponential leads to a non-factorizable character of the distribution function (18).

Using the identity $\exp _{q-1}[z]=\exp \left\{\ln \exp _{q-1}[z]\right\}$ and the new variable $z_{q}=\ln \exp _{q-1}[z] \equiv(q-1)^{-1} \ln (1+(q-1) z)$, one can formally rewrite $q$ exponential in the usual exponential form, $\exp _{q-1}[z]=\exp \left[z_{q}\right]$. This allows 
to reformulate any $q$-exponential distribution function, e.g., (18), in a factorizable form with respect to $z_{q}$. Consequently, one can formally achieve an additive character of Tsallis formalism with respect to such " $q$-deformed " entities $z_{q}$.

Almeida [9] has derived Tsallis distribution (18) from the first principles of statistical mechanics using the parametrization

$$
\frac{d \theta}{d E}=q-1
$$

where $\theta \equiv 1 / \beta$ is the statistical temperature. Tsallis parameter $q$ is given a physical interpretation in terms of heat capacity of the heat bath due to the fact that $\theta$ is related to the temperature of the heat bath. For an infinite heat capacity (thermostat), $q=1$, one recovers canonical Gibbs distribution while for finite heat capacity (quasithermostat), $q \neq 1$, one gets power law distributions.

The microcanonical approach meets this viewpoint as it establishes dependence of $q$ on the number of particles $M$ of the quasithermostat which evidently characterizes its heat capacity, $d \theta / d E \sim 1 / M$. It is worthwhile to note however that in general the form of the dependence $\theta(E)$ in the l.h.s. of Eq. (27) seems to be not fixed by first principles of statistical mechanics. The linear dependence, $\theta(E)=(q-1) E$, does yield Eq. (27) while a quadratic dependence would require yet another parameter. In contrast, the used microcanonical approach with the kinetic energy in the form (17) suggests only one parameter, $M$, which we relate to $q$ via Eq. (17).

\section{The entropy}

The entropy associated to the distribution function $f_{N}=f_{N}(H(x, a), \beta, q)$ defined by (18) can be readily obtained, up to a factor and constant term.

First, we identically rewrite Eq. (18) as

$$
f_{N}=\exp _{q-1}\left[\beta F_{q}-\left(\beta-(q-1) F_{q}\right) H(x, a)\right],
$$

where we have denoted

$$
F_{q}=\beta^{-1} \ln _{q-1} Z_{q}^{-1},
$$

and $q$-logarithm is an inversion of $q$-exponential, $\ln _{s}[z]=\left(z^{s}-1\right) / s, s=q-1$. 
Some useful formulas with $q$-logarithm are:

$$
\begin{gathered}
\lim _{q \rightarrow 1} \ln _{q-1} z=\ln z, \quad \ln _{q-1} z^{-1}=-\ln _{1-q} z \\
\ln _{q-1} z^{p}=p \ln _{p(q-1)} z, \quad z \ln _{q-1} z=z^{q} \ln _{1-q} z \\
d \ln _{q-1} z / d z=z^{q-2}=z^{-1}\left(1+(q-1) \ln _{q-1} z\right), \\
\int \ln _{q-1} z d z=q^{-1} z\left(\ln _{q-1} z-1\right),
\end{gathered}
$$

and

$$
\ln _{q-1} z_{1} z_{2}=\ln _{q-1} z_{1}+\ln _{q-1} z_{2}+(q-1) \ln _{q-1} z_{1} \ln _{q-1} z_{2},
$$

Then, with the use of the inverse function,

$$
H=\rho\left(f_{N}\right)=\left(\beta F_{q}-\ln _{q-1} f_{N}\right) /\left(\beta-(q-1) F_{q}\right),
$$

the normalization condition, $\int f_{N} d x=1$, the thermodynamic heat, $\delta Q=$ $\int H(x, a)\left(\delta f_{N}\right) d x$, and the integrability condition, $d Q=T(\beta) d S_{q}$ one can obtain (cf. [9])

$$
S_{q}(a, T)=-\int f_{N} \ln _{q-1} f_{N} d x \equiv-\int f_{N}^{q} \ln _{1-q} f_{N} d x .
$$

Here, the representation

$$
S_{q}=T^{-1}(\beta) \int_{0}^{f_{N}}(\rho(z)+\text { const }) d z d x
$$

with a suitable constant has been used. The integrability factor is found to be $T(\beta)=\left(q \beta-q(q-1) F_{q}\right)^{-1}$.

With $\beta^{-1}=k T$, we obtain

$$
\delta Q=\left(\frac{q}{k T}-q(q-1) F_{q}\right) d S_{q} .
$$

In the discrete case characterized by microstates with energies $E_{n}$, the obtained quasicanonical distribution function (18) and the entropy (36) are written as

$$
\begin{gathered}
f_{n}=\frac{1}{Z_{q}} \exp _{q-1}\left[-\beta E_{n}\right], \\
Z_{q}=\sum_{n=1}^{W} \exp _{q-1}\left[-\beta E_{n}\right], \quad \sum_{n=1}^{W} f_{n}=1,
\end{gathered}
$$




$$
\begin{gathered}
S_{q}=-\sum_{n=1}^{W} f_{n} \ln _{q-1} f_{n} \equiv-\sum_{n=1}^{W} f_{n}^{q} \ln _{1-q} f_{n} \\
\equiv-\frac{\sum_{n=1}^{W}\left(f_{n}^{q}-f_{n}\right)}{q-1}=-\frac{\sum_{n=1}^{W} f_{n}^{q}-1}{q-1} .
\end{gathered}
$$

The entropy (41) coincides with Tsallis entropy [1], with $q$ treated as an entropic parameter, and reduces to Boltzmann-Gibbs-Shannon entropy, $S=-\sum f_{n} \ln f_{n}$, at $q \rightarrow 1$. It is remarkable to note that either $f_{n}$ and $f_{n}^{q}$ can be used for the averaging in Eq. (41) provided a suitable choice of $q$.

It is well known that by extremizing the (convex) entropy (41) under suitable constraints [3] one obtains Tsallis distribution (39).

Due to the property (34) of $q$-logarithm the entropy (41) is non-additive,

$$
S_{q}(A, B)=S_{q}(A)+S_{q}(B \mid A)+(q-1) S_{q}(A) S_{q}(B \mid A),
$$

where we use the conditional entropy for statistically dependent systems $A$ and $B$ (e.g., subsystems with long-range interactions) based on the conditional distribution function $f(A, B)=f(A \mid B) f(B)=f(B \mid A) f(A)$.

In the case $A$ and $B$ are statistically independent in a conventional sense (e.g., subsystems with short-range interactions), we have formally $S_{q}(A \mid B)=$ $S_{q}(A)$ and $S_{q}(B \mid A)=S_{q}(B)$.

We note that the $(q-1)$ term in Eq. (42) is non-zero even for such "statistically independent" systems contrary to a naive expectation that the statistical independence should imply an additive character of the entropy $S_{q}$. The origin of this implication seems to be due to the fact that here the parameter $q$ does not depend by construction on a scale or correlation parameter, such as a correlation length, which allows one to determine a statistical independence of the systems $A$ and $B$ in a physical context (when the systems $A$ and $B$ are separated by distances much bigger than the correlation length their evolutions are physically independent).

Formally, for a constant parameter $q \neq 1$ the systems appear to be always statistically dependent on each other. Deeper analysis is required for a consistent formulation of the statistical independence within the framework of non-additive formalism (42). 


\section{Normal random momenta and fluctuating statistical temperature}

Recently, Beck [5] have studied application of Tsallis formalism to turbulent flows and indicated that the factor of Tsallis equilibrium distribution function (39) can be obtained by averaging Boltzmann factor of the canonical Gibbs distribution [6],

$$
\int_{0}^{\infty} e^{-\tilde{\beta} E_{n}} f(\tilde{\beta}) d \tilde{\beta}=\left(1-(q-1) \beta E_{n}\right)^{\frac{1}{q-1}},
$$

where $1-q=2 / m$ and

$$
f(\tilde{\beta})=\frac{1}{\Gamma\left(\frac{m}{2}\right)}\left(\frac{n}{2 \beta}\right)^{\frac{m}{2}} \tilde{\beta}^{\frac{m}{2}-1} \exp \left[-\frac{m \tilde{\beta}}{2 \beta}\right]
$$

is the probability density of the $\chi^{2}$ distribution of order $m$ (the distribution of sum of squares of $m$ normal random variables with mean zero and unit variance), with the average $\langle\tilde{\beta}\rangle \equiv \int_{0}^{\infty} \tilde{\beta} f(\tilde{\beta}) d \tilde{\beta}=\beta$ and the relative variance $\left(\left\langle\tilde{\beta}^{2}\right\rangle-\langle\tilde{\beta}\rangle^{2}\right) /\langle\tilde{\beta}\rangle^{2}=2 / \mathrm{m}$. This is viewed as the result of fluctuation of temperature or fluctuation of energy dissipation [7, 8] and has been found of particular relevance for nonequilibrium systems. Beck have used an assumption that the time scale on which $\tilde{\beta}$ fluctuates is much bigger than that of kinetic relaxation of the system. This provides a quasistatic behavior of the system with respect to variation of $\tilde{\beta}$.

We see that $\chi^{2}$ randomization and averaging of the canonical Gibbs distribution over random $\tilde{\beta}$ leads to Tsallis distribution.

It seems that the relevance of $\chi^{2}$ distribution (44) to relate Boltzmann and Tsallis factors is not due to a coincidence. By comparing this approach [5, 6] with the above obtained results (17) and (18), we see that the number of random variables is $m=3 M$, which is exactly the total number of components of three-dimensional momenta $p_{i}$ of $M$ particles constituting the quasithermostat. Since the quasithermostat is assumed to be in a quasi-equilibrium state we can treat the momenta $\vec{p}_{i}$ (microscopic characteristics) to be normal random variables with mean zero and unit deviation as a simplest model. Hence, the kinetic energy (7) is $\chi^{2}$ distributed due to its definition. We associate the kinetic energy of particles in quasithermostat to 
its statistical temperature $\beta^{-1}$ in accord to Eq. (13). Hence, $\tilde{\beta}^{-1}$ becomes $\chi^{2}$ random variable.

This partially clarifies the physical origin of the $\chi^{2}$ distribution used by Beck in Eq. (43). However, we note that in our approach $\chi^{2}$ distribution arises for the statistical temperature $\tilde{\beta}^{-1}$ (not for the inverse statistical temperature $\tilde{\beta}$ ).

Usually, normal (not $\chi^{2}$ ) distribution of fluctuating temperature with the relative variance from Eq. (58) is used as a good approximation. We note that the averaging of the canonical Gibbs distribution over normally distributed $\tilde{T}$ (i.e., $\tilde{\beta}^{-1}$ ) with the mean $T$ and some deviation $\sigma$ is not analytically tractable, while the averaging over normally distributed $\tilde{\beta}$ yields the analytic result,

$$
\int_{0}^{\infty} e^{-\tilde{\beta} H} g(\tilde{\beta}) d \beta=e^{-\beta H+\frac{1}{2} \sigma^{2} H^{2}}\left(1-\operatorname{erf}\left(\frac{\sigma^{2} H-\beta}{\sqrt{2} \sigma}\right)\right)
$$

where

$$
g(\tilde{\beta})=\frac{1}{\sigma \sqrt{\pi / 2}} \exp \left[-\frac{(\tilde{\beta}-\beta)^{2}}{2 \sigma^{2}}\right]
$$

is normal distribution, and $\operatorname{erf}(x)$ is the error function. The resulting expression (45) includes one special function and is less handful as compared with Tsallis distribution (43).

Since the entropy $S_{q}$ and mean energy $\bar{H}$ of the equilibrium system are not additive for $q \neq 1$, the function $F_{q}$ is not additive as well. Indeed, from Eqs. (18) and (36), we have

$$
S_{q}=\left(\beta-(q-1) F_{q}\right) \bar{H}-\beta F_{q},
$$

where $\bar{H}=\int H f_{N} d x$. This implies that $\beta$ is not a universal parameter of the system, in contrast to the case of additive entropy and mean energy. Indeed, $\beta$ is not the only parameter characterizing the quasithermostat, with $q$ being the second temperature parameter measuring the relative variance of the fluctuating parameter around the value $\beta$ viewed as an average.

From the viewpoint of canonical Gibbs distribution, accounting for a finiteness of thermostat made in Sec. 2 automatically arises to the averaged $\chi^{2}$ distributed fluctuation of the parameter $\tilde{\beta}$ around the mean $\beta$ with the relative variance $\sim 1 / M$. Actually we associate the fluctuations of temperature with eventual fluctuations of the energy of a finite thermostat. We conclude that the $\chi^{2}$ statistics is implicit in Tsallis statistical mechanics. 
The spatial scale dependence of the entropic parameter, $q=q(r)$, introduced by Beck [5] can be readily understood since $q$ is related to the number of particles. For bigger spatial scale the fluctuations become smaller so that $q \rightarrow 1$ in the big scale asymptotics.

To illustrate how the resulting nonadditivity affects the temperature characteristics, we consider two systems with Hamiltonians $H_{1}$ and $H_{2}$, respectively, at the same $\beta$. Abe and Rajagopal [12] have elaborated the power-low distribution based thermodynamics of equilibrium to much extent. Below, we discuss this issue in the context of fluctuations of the parameter $\beta$.

For weakly interacting canonical systems we have $\exp \left[-\beta H_{1}\right] \exp \left[-\beta H_{2}\right]=$ $\exp [-\beta H]$, where $H=H_{1}+H_{2}$ is conserved. As usual, this means that the two equilibrium systems compose an equilibrium system at the same $\beta$, while at different $\beta$ there is no equilibrium, with conserved $H$. The $\chi^{2}$ randomization, $\beta \rightarrow \tilde{\beta}$, and averaging of each of the two canonical systems, with the same average $\beta$ and relative variance $q-1$, gives us $\exp _{q-1}\left[-\beta H_{1}\right] \exp _{q-1}\left[-\beta H_{2}\right]=\exp _{q-1}\left[-\beta H_{\text {eff }}\right]$, where we have denoted $H_{\text {eff }}=$ $H+(q-1) \beta H_{1} H_{2}$. On the other hand, the averaging of the composite system yields a different result, $\left\langle\exp \left[-\beta\left(H_{1}+H_{2}\right)\right]\right\rangle_{\beta}=\exp _{q-1}[-\beta H]$.

It is misleading to interpret the above relations as that the composite system appears to be not at thermal equilibrium despite the identical $\beta$. A thermal equilibrium of two systems in the sense of their absolute temperatures is well formulated for a non-fluctuating $\beta$ but not for the average, $\beta$, of

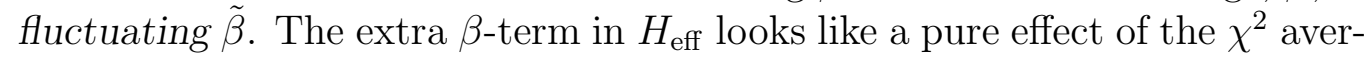
aging since it is proportional to the relative variance $q-1$. Indeed, in general $\left\langle f\left(\tilde{\beta}, H_{1}\right)\right\rangle_{\beta}\left\langle f\left(\tilde{\beta}, H_{2}\right)\right\rangle_{\beta} \neq\left\langle f\left(\tilde{\beta}, H_{1}\right) f\left(\tilde{\beta}, H_{2}\right)\right\rangle_{\beta}$ regardless to factorizability of the function $f(\tilde{\beta}, H)$ with respect to additive $H$. Even more difficulties in understanding thermal equilibrium would arise when one naively considers two systems with the same average $\beta$ and different relative variances, $q_{1}-1$ and $q_{2}-1$.

In order to clarify the physical content of the problem of fluctuating $\tilde{\beta}$ we turn to the time scale hierarchy emerging from the microscopic consideration of a dilute gas and weakly inhomogeneous systems of a finite characteristic size. 


\section{The time scale hierarchy}

Internal characteristics of a dilute gas with density $n=N / V$ are known to be the interaction range $r_{\text {int }}$, the mean distance between particles, $r_{\text {ave }} \simeq n^{-1 / 3}$, and the free-flight length $r_{\text {fli }} \simeq 1 /\left(n r_{\text {int }}^{2}\right)$. The main parameter of a dilute gas is the density parameter,

$$
\varepsilon \equiv n r_{\text {int }}^{3} \ll 1 \text {. }
$$

For example, ambient air is characterized by the typical value $\varepsilon \simeq 10^{-4}$. For small $\varepsilon$ we have scale hierarchy,

$$
r_{\text {int }} \ll r_{\text {ave }} \ll r_{\text {fli }},
$$

with $r_{\text {int }}^{3}: r_{\text {ave }}^{3}: r_{\text {fli }}^{3}=1: \varepsilon^{-1}: \varepsilon^{-3}$, from which it follows the time scale hierarchy,

$$
\tau_{\text {int }} \ll \tau_{\text {ave }} \ll \tau_{\text {fli }},
$$

where $\tau_{\text {int }}=r_{\text {int }} / v$ is the interaction time, $\tau_{\text {ave }}=r_{\text {ave }} / v$ is the average time, $\tau_{\text {fli }}=r_{\text {fli }} / v$ is the free-flight time, and $v$ is some characteristic velocity of particles. Typically, for dilute gases $\tau_{\text {int }} \simeq 10^{-12}$ sec and $\tau_{\text {fli }} \simeq 10^{-8}$ sec.

For such systems the quasi-closeness and quasi-equilibrium of subsystems are well defined, and a description in terms of one- and two-particle probability distribution functions, or mean local densities (particle number, momentum, and energy), is rigorously justified.

One-particle distribution function varies much slower than the higher order ones due to the smallness of $\varepsilon$, providing thus quasi-conservation of the mean local densities. At the microscopic level a quasi-additivity of the (quasi)local energy density (hence, of the energy) can be seen from the last term in its definition,

$$
h(\vec{r})=\sum_{i} \delta\left(\vec{r}-\vec{r}_{i}\right)\left[\vec{p}_{i}^{2} / 2 m+\sum_{j \neq i} \Phi\left(\left|\vec{r}_{i}-\vec{r}_{j}\right|\right)\right] .
$$

Here the last sum makes the biggest contribution for the coordinates $\vec{r}_{j}$ of particles which are at distances less or about $r_{\text {int }}$ from the point $\vec{r}$. In fact, whether $h(\vec{r})$ can be taken additive or non-additive depends on the scale.

We start by considering a sequence of systems,

$$
\mathcal{N}_{1} \subset \mathcal{N}_{2} \subset \cdots \subset \mathcal{N}_{i} \subset \cdots \subset \mathcal{N}_{\infty}
$$

generated by the relations between the numbers of particles in these systems, $1 \ll N_{1} \ll N_{2} \ll \cdots \ll N_{i} \ll \cdots \ll N_{\infty}=\infty$. The systems $\mathcal{N}_{1}$ and $\mathcal{N}_{\infty}$ can 
be viewed as a smallest system and an ideal thermostat, respectively, in the equilibrium state. More specifically, the systems $\mathcal{N}_{i}$ are treated as thermodynamical systems of characteristic sizes $r_{i}$, which are external characteristics, such that

$$
\begin{array}{r}
r_{\text {int }} \ll r_{\text {ave }} \ll r_{1} \ll \cdots \ll r_{k-1} \ll r_{\text {fli }} \ll \cdots \\
\cdots \ll r_{k} \ll \cdots \ll r_{\infty}=\infty
\end{array}
$$

Here, we have used the fact that for a dilute gas the free-flight length is much bigger than the interaction range, $r_{\mathrm{fli}} \gg r_{\text {int }}$, so that some number $(k-1)$ of systems fall into the range between $r_{\text {ave }}$ and $r_{\text {fli }}$. In this paper, we will not consider such systems which require a separate study.

Actually we assume that $\mathcal{N}_{\infty}$ can be represented as a collection of weakly interacting equilibrium systems $\mathcal{N}_{1}$ so that all bigger systems $\mathcal{N}_{i}, i>1$, are in incomplete equilibrium state at some time scale.

We remind that in the framework of the canonical Gibbs distribution, the interaction between different $\mathcal{N}_{1}$ 's is taken negligible, $\xi \equiv H_{\text {int }} / H_{N_{1}}=0$ (zero order approximation in small $\xi)$. However, the relaxation of some system $\mathcal{N}_{i}$, $i>1$, to an equilibrium state is solely related to $H_{\text {int }}$ so that at least first order approximation in small $\xi$ should be used to describe the relaxation process.

The kinetic relaxation length $r_{\text {rel }} \sim r_{\text {fli }}$, and the associated kinetic relaxation time, $\tau_{\text {rel }} \sim \tau_{\text {fli }}$, are much bigger than the correlation length, $r_{\text {cor }} \sim r_{\text {int }}$ and the associated correlation time, $\tau_{\text {cor }} \sim \tau_{\text {int }}$, for $\varepsilon \ll 1$.

In terms of small parameter $\varepsilon$, one can define (see e.g. [13])

$$
r_{1}=\sqrt{\varepsilon} r_{\mathrm{fli}}, \quad \tau_{1}=\sqrt{\varepsilon} \tau_{\mathrm{fli}}, \quad N_{1}=1 / \sqrt{\varepsilon},
$$

for the smallest system $\mathcal{N}_{1}$. The size $r_{1}=v \tau_{1}$ is taken to provide conservation of the local densities of particle number, momenta and energy in the approximation of weak inhomogeneity,

$$
r_{\text {cor }} / r_{1} \ll 1
$$

at the time scale $\tau_{1}$. For $\varepsilon=10^{-4}$, we obtain the minimal number of particles $N_{1}=100$. To specify the hierarchy, we can put $N_{i}=1 / \varepsilon^{i / 2}$.

To each characteristic size $r_{i}, i>k$, we can associate the gas dynamical relaxation time,

$$
\tau_{i}=\tau_{1}\left(r_{i} / r_{\mathrm{fli}}\right)^{2}, \quad i>1
$$


so that the time scale hierarchy is

$$
\tau_{\text {int }} \ll \tau_{\text {ave }} \ll \tau_{\text {fli }} \ll \tau_{k} \ll \tau_{k+1} \cdots \ll \tau_{\infty}=\infty .
$$

For $i \geq k$, the sizes of the systems $\mathcal{N}_{i}$ are much bigger than $r_{\text {fli }}$ so that for such systems one more small parameter can be introduced, $K_{i} \equiv r_{\text {fli }} / r_{i}$, Knudtsen parameter, in terms of which $\tau_{i}=\tau_{1} / K_{i}^{2}$.

It should be emphasized that due to $N_{1}=1 / \sqrt{\varepsilon}$ the $\varepsilon=0$ approximation in the (nonfluctuating) canonical Gibbs distribution implicitly means that $N_{1}=\infty$ (the approximation of a continuous medium for $N_{1}$ particle system). Evidently, the consideration of bigger systems $\mathcal{N}_{i}, i>1$, becomes of no sense in the $\varepsilon=0$ approximation. This is the point where a large scale dependence is totally ignored within the approximation of nonfluctuating distribution function. To keep this dependence one should derive the quasi-equilibrium distribution function in at least the first order approximation in $\varepsilon$ and allow distribution function to fluctuate.

\section{Fluctuating probability distribution func- tions}

Now we turn to fluctuations. Following to Onsager principle, kinetics of a linear non-equilibrium thermodynamical system (its relaxation to an equilibrium state) defines fluctuations in the system. In turn, the fluctuations of the thermodynamical entities (evolution of the time correlation functions of their deviations from equilibrium values) can be equivalently described in terms of Langevin equation with $\delta$-correlated stochastic sources. Thus the fluctuations of equilibrium systems can be viewed as a stationary process with the time scales defined by the relaxation times $\tau_{i}, i>k$, of the gas dynamical stage of the relaxation. As shown in Sec. 5 these time scales are known to be much bigger than that of the kinetic relaxation $\tau_{\text {rel }}$ thus providing a local equilibrium framework.

We are interested in a finite thermostat so that we take some finite size equilibrium system $\mathcal{N}_{j}$ serving as a quasithermostat for the system $\mathcal{N}_{i}$, i.e., we assume $N_{j} \gg N_{i}$. In accord to the above consideration, the time scale $\tau_{j}$ of the quasithermostat $\mathcal{N}_{j}$ at which its $\beta$ (kinetic energy per particle) fluctuates is much bigger than the time scale $\tau_{i}$ at which any thermodynamical entity of the system $\mathcal{N}_{i}$ fluctuates. In general, there is finite number of big systems 
$\mathcal{N}_{i+1}, \mathcal{N}_{i+2}, \ldots, \mathcal{N}_{j}$, each making contribution to fluctuation of the temperature with different relative variances at corresponding time scales $\tau_{i+1}, \ldots, \tau_{j}$. Observed marginal distribution for $\mathcal{N}_{i}$ will depend on the observation time $\tau_{\text {obs }}$ as compared to these time scales. We remark that the observed relative variance of fluctuating $\beta$ is thus depends on the scale and $\tau_{\text {obs }}$.

At the time scale $\tau_{\text {obs }} \sim \tau_{i}$ the canonical Gibbs distribution makes a very good description of $\mathcal{N}_{i}$, with $\beta$ treated as a constant, and an additive character of the entropy and mean energy.

At long time scale, $\tau_{\text {obs }} \geq \tau_{j} \gg \tau_{i}$, the fluctuations of $\beta$ can not be ignored so that Tsallis-like distribution emerges as an effective description, i.e., we should replace $\beta \rightarrow \tilde{\beta}$, and make the averaging $\left\langle e^{-\tilde{\beta} H_{i}}\right\rangle_{\beta}$, with $\beta=\langle\tilde{\beta}\rangle$ and some relative variance. This results in a non-additive character of the entropy and mean energy of $\mathcal{N}_{i}$. Note however that in the latter case the usual quasi-equilibrium (thermal equilibrium) still holds for any given value of $\tilde{\beta}$ at the small time scale $\tau_{i}$. This picture meets that assumed by Beck [5].

For $j \rightarrow \infty$ (an ideal thermostat), we have $\tau_{j} \rightarrow \infty$ so that $\beta$ is constant at any time scale $\tau>\tau_{i}$, thus $q=1$ for $\mathcal{N}_{\infty}$.

To put the above consideration on solid grounds, below we outline statistical mechanics approach to fluctuations, within a dynamical framework.

We can treat the quasithermostat $\mathcal{N}_{j}$ as an equilibrium system, which can be described in pure additive thermodynamical terms, with the canonical Gibbs distribution, $Z^{-1} \exp \left[-H_{j} / k T\right]$, providing the averaging in $\mathcal{N}_{j}$.

However, kinetic and thermodynamic fluctuations naturally occur in the quasi-equilibrium system $\mathcal{N}_{j}$ and can be calculated in a linear approximation (small fluctuations) for the case of weak inhomogeneity of the system, Eq. (55). We notice that rigorous description of fluctuations is out of scope of the canonical Gibbs distribution and the one-particle distribution function entering Boltzmann equation.

In a dynamical framework, one should consider Bogolyubov equations with a short-range interaction potential, $\Phi\left(\left|\vec{r}_{m}-\vec{r}_{n}\right|\right)$, for point-like particles (providing convergence of the integral for local energy density), small density parameter $\varepsilon$ (i.e., three- and higher order particle collisions are ignored), and weak inhomogeneity (providing small gradient of the one-particle distribution function), and account for the two-particle correlation function with a partial relaxation of initial correlations. This implies fluctuations of the one-particle distribution function; see e.g. [13] and review [14]. The form of the resulting kinetic equations allows one to study separately implications related to Brownian effect (a discrete collision structure of the medium) thus 
discarding influence of fluctuations on kinetic processes.

The resulting picture is equivalent to consideration of some Langevin equations with $\delta$-correlated stochastic sources, from which one can extract two-point correlation functions and spectral densities of the gas density, velocity, and pressure, for a local equilibrium state. In the local equilibrium approximation correlation functions of the stochastic sources can be found also by phenomenological methods of non-equilibrium thermodynamics 15.

The spectral densities can be used to estimate the gas dynamical relaxation time, $1 / \tau \sim \nu \vec{k}^{2} \sim \kappa \vec{k}^{2}$, where $\nu$ is kinematic viscosity, $\kappa$ is heat conductivity, and $\vec{k}$ is wave vector. For a finite system of characteristic size $L$, the wave vector $\vec{k}$ is naturally bounded from below, $|\vec{k}| \geq 1 / L$, from which with the use of estimation $\nu \sim \kappa \sim v r_{\text {fli }}$ it follows Eq. (56).

We focus on the temperature fluctuations, for which one can obtain the approximate variance,

$$
\left\langle(\Delta \tilde{T})^{2}\right\rangle \simeq k T^{2}(d T / d E)_{V}
$$

with temperature $\tilde{T}$ being normally distributed (in this approximation), and $T$ in the r.h.s. is given in terms of equilibrium value. This means that $\tilde{T}$ fluctuates about the average $T$ with the relative variance $k / C_{v}$, where $C_{v}=(d E / d T)_{V} \sim N_{j}$ is a heat capacity of the quasithermostat $\mathcal{N}_{j}$, thus ending up with the consideration of Sec. 4 .

It is worthwhile to note that the entropy $S$ of $\mathcal{N}_{j}$ fluctuates as well, $\left\langle(\Delta \tilde{S})^{2}\right\rangle \simeq k C_{p}, C_{p} \sim N_{j}$.

Clearly, natural fluctuations occur not only in the quasithermostat $\mathcal{N}_{j}$ but also in the considered smaller system $\mathcal{N}_{i}$. However, we can ignore these fluctuations as they make small contribution to specific fluctuation of the temperature $\tilde{T}$ governed by the quasithermostat, due to $N_{i} \ll N_{j}$.

We point out that a major difference from the above consideration arises when turning to fully developed turbulence considered in [5]. Beck used statistical temperature related to kinetic coefficients (fluctuations of which are evidently not related to a finiteness of quasithermostat) and have averaged the probability distribution function (describing a stationary non-equilibrium state) derived from a nonlinear Langevin equation which is different from the canonical Gibbs distribution. Fluctuations of the friction coefficient (or viscosity) are seem to be of a kinetic type originating from large scale fluctuations of the phase-space density function averaged over the smallest scale. Such fluctuations may have a big relative variance as they are not related 
to the particle number of some big system, with the main contribution to the energy dissipation happening at the smallest scale being fluctuations, $\tilde{u}_{i} \sim \delta u_{i}$, where $\tilde{u}_{i}$ is velocity and $\delta u_{i}$ is fluctuation of the velocity. Indeed, for Kolmogorov (the smallest) length scale $\eta$ of the turbulent flow Beck has derived under simple assumptions a theoretical value of the entropic parameter, $q=3 / 2$, which essentially deviates from unity (the relative variance is considerable, $q-1=1 / 2$ ). One can see [5] that Tsallis formalism does work in this case as well. For bigger scales, fluctuations of the energy dissipation decrease so that in zero order approximation in $\eta / r$ the energy dissipation is constant. Clearly, one should assign the value $q=1$ for this large scale asymptotics.

Fluctuations of $\tilde{\beta}$ in the canonical Gibbs distribution mean that we deal with a fluctuating distribution function. The $\chi^{2}$ average value of this fluctuating distribution function is Tsallis distribution,

$$
\left\langle e^{-\tilde{\beta} H}\right\rangle_{\beta}=e_{q-1}^{-\beta H} .
$$

The relative variance of this fluctuation is

$$
\frac{\left\langle e^{-2 \tilde{\beta} H}\right\rangle_{\beta}-\left\langle e^{-\tilde{\beta} H}\right\rangle_{\beta}^{2}}{\left\langle e^{-\tilde{\beta} H}\right\rangle_{\beta}^{2}}=s \ln _{s}\left(1+\frac{s^{2} \beta^{2} H^{2}}{1+2 s \beta H}\right),
$$

where $s=1-q$. For small $(1-q)$, this relative variance behaves as $(1-q)^{3}$. Also, up to a first order in $(q-1)$, we obtain

$$
\exp _{q-1}[-\beta H] \simeq\left(1+\frac{q-1}{2} \beta^{2} H^{2}\right) \exp [-\beta H]
$$

It is remarkable to note that this function is non-factorizable with respect to $H$ because of the presence of $H^{2}$.

From the above dynamical consideration it follows that fluctuations of a probability distribution function arise naturally in studying both equilibrium and non-equilibrium phenomena. However, in studying equilibrium state one usually uses non-fluctuating distribution function, thus discarding specific effects such as wellknown Brownian one. Namely, the $\varepsilon=0$ approximation means a continuous medium approximation, at which Brownian motion can not be described, even for the smallest physical equilibrium system $\mathcal{N}_{1}$ consisting of, e.g., $N_{1}=100$ particles for $\varepsilon=10^{-4}$.

In contrast, the use of a phenomenological Tsallis approach makes possible to account for some of the effects related to natural fluctuations of 
an equilibrium distribution function or that corresponding to a stationary non-equilibrium process.

Finally, we point out that even small fluctuations of the one-particle distribution function, $\delta f / f \ll 1$, make an essential contribution (as big as Boltzmann integral which is treated as a leading term) to Boltzmann equation with Langevin sources [13]. This indicates importance of accounting for fluctuations of probability distribution functions, both for equilibrium and non-equilibrium states, at some scales.

\section{Discussion}

The microcanonical approach in the form presented in this paper evidently can not cover a universal character of Tsallis formalism. First, since we assume $M \gg 1$ the parameter $q-1$ is very close to zero that allows us to use expansion in $q-1$ and calculate small corrections to the canonical Gibbs distribution, while for considerable $q-1$ the arguments of our derivation in Sec. 2 break down. Second, we have ignored the interaction energy $H_{\text {int }}$ that is not valid for systems with long-range microscopic interactions to which Tsallis statistics is applied. Also, the nonextensivity can arise not only as the effect of a finite heat bath. For example, Tsallis distribution arises in the description of polydispersed colloids with the fluctuating parameter being particle size [6], and was used to describe statistical properties of fully developed turbulence [7, 8]; see also review in [3].

In general, fluctuations of the parameter $\beta$ (or statistical temperature) and averaging of the fluctuating distribution function over $\chi^{2}$-like distribution can be viewed as a defining feature of Tsallis-like nonextensive statistical distributions, regardless to the origin of fluctuations.

Adopting this viewpoint, one can try various types of distribution of $\tilde{\beta}$ in replace of $\chi^{2}$ distribution, and allow considerable relative variance of $\tilde{\beta}$. For example, it is natural to assume Poisson distribution for the random momenta $\vec{p}_{i}$ in replace of the normal distribution; see also [16]. Also, one may be interested in a more detailed description and account for higher order momenta such as biquadratic deviations of $\tilde{\beta}$.

From this point of view, Tsallis formalism can be treated as a suitable universal tool to deal with fluctuating distribution functions without turning to underlying kinetic issues. However, it should be noted that the use of an averaged fluctuating distribution function means incomplete statistical 
description.

Finally, we stress that the very notions of extensivity (additivity) and intensivity in thermodynamics are essentially based on the requirement that the system is homogeneous, which is provided for big systems with weak interactions or, more precisely, in the thermodynamic limit, $N, V \rightarrow \infty$, $N / V=$ const. These notions make no strict sense for inhomogeneous systems such as finite systems or systems characterized by the size of the order of correlation length $r_{\text {cor }}$. As a consequence, in this case there is no thermodynamical equivalence between equilibrium microcanonical and canonical ensembles.

\section{References}

[1] C. Tsallis, J. Stat. Phys. 52, 479 (1988).

[2] C. Tsallis, R.S. Mendes, and A.R. Plastino, Physica 261A, 534 (1998).

[3] C. Tsallis, Entropic nonextensivity: a possible measure of complexity, cond-mat/0010150.

[4] S. Abe, Phys. Lett. 271A, 74 (2000); S. Abe, S. Martinez, F. Pennini, A. Plastino, Phys. Lett. 281A, 126 (2001); S. Abe, Phys. Rev. 63E, 061105 (2001); S. Abe and A.K. Rajagopal, Nonuniqueness of canonical ensemble theory arising from microcanonical basis, quantph/9911097; Nonadditive conditional entropy and its significance for local realism, quant-ph/0001085; Justification of power-law canonical distributions based on generalized central limit theorem, cond-mat/0003380; A.K. Rajagopal and S. Abe, Phys. Rev. Lett. 83, 1711 (1999); Statistical mechanical foundations for systems with nonexponential distributions, cond-mat/0003493.

[5] C. Beck, Generalized statistical mechanics and fully developed turbulence, cond-mat/0110073.

[6] R. Johal, An interpretation of Tsallis statistics based on polydispersity, cond-mat/9909389.

[7] C. Beck, Physica 277A, 115 (2000); Phys. Lett. 287A, 240 (2001); Dynamical foundations of nonextensive statistical mechanics, to appear in 
Phys. Rev. Lett. (2001); Non-additivity of Tsallis entropies and fluctuations of temperature, cond-mat/0105371.

[8] G. Wilk and Z. Wlodarczyk, Phys. Rev. Lett. 84, 2770 (2000).

[9] M. P. Almeida, Generalized entropies from first principles, condmat/0102112.

[10] A. Plastino; A. R. Plastino, Brazilian J. of Phys. 29 , no. 1 (1999).

[11] A.K. Aringazin, Phys. Lett. 314B, 333 (1993); A.K. Aringazin, V.V. Arkhipov, and A.S. Kudusov, BRST approach to Hamiltonian systems, hep-th/9811026.

[12] S. Abe and A.K. Rajagopal, Macroscopic thermodynamics of equilibrium characterized by power-law canonical distributions, cond-mat/0009400.

[13] Yu.L. Klimontovich, Statistical Physics (Nauka, Moscow, 1982) (in Russian).

[14] S.V. Gantsevich, V.L. Gurevich, R. Katilius, Rev. Nuovo Cimento 2, 1 (1979).

[15] E.M. Lifshitz and L.P. Pitaevski, Statistical Physics, Part 2. Nauka, Moscow, 1978. (in Russian).

[16] F. Sattin and L. Salasnich, Multi-parameter generalization of nonextensive statistical mechanics, physics/0110055. 